Research paper

\title{
Development of a microelectrochemical biosensor for the real-time detection of choline
}

\author{
Keeley L. Baker*, Fiachra B. Bolger, John P. Lowry \\ Neurochemistry Research Unit, BioAnalytics Laboratory, Department of Chemistry, Maynooth University, Maynooth, Co. Kildare, Ireland
}

\section{A R T I C L E I N F O}

\section{Article history:}

Received 21 August 2016

Received in revised form 9 November 2016

Accepted 22 November 2016

Available online 23 November 2016

\section{Keywords:}

Acetylcholine

Choline

Biosensor

Oxygen interference

Selectivity

Sensitivity

\begin{abstract}
A B S T R A C T
Here we describe the development of a first generation biosensor for the detection of brain extracellular choline, investigating important considerations for in-vivo monitoring such as sensor sensitivity, $\mathrm{O}_{2}$ interference and selectivity. Extensive optimisation of choline biosensor designs resulted in a biosensor with excellent sensitivity towards choline $(0.54 \pm 0.03 \mathrm{nA} / \mu \mathrm{M})$. Oxygen interference studies demonstrate a $1 \%$ reduction in current at $50 \mu \mathrm{M} \mathrm{O}_{2}$ when compared to atmospheric $\mathrm{O}_{2}$ levels $(200 \mu \mathrm{M})$, indicating that the sensor can be used for reliable choline monitoring, free from changes in current associated with physiological $\mathrm{O}_{2}$ fluctuations. A negligible sensitivity of $0.0021 \pm 0.0002 \mathrm{nA} / \mu \mathrm{M} \mathrm{n}=8$ was achieved utilising poly-phenylenediamine (PPD) as a permselective membrane for interference rejection of ascorbic acid (AA), the most physiologically important endogenous electroactive species present in the brain. The optimised biosensor when implanted into the striatum of a freely moving rat successfully detected local perfusions of choline demonstrating the sensors ability to detect choline in-vivo.
\end{abstract}

(C) 2016 Elsevier B.V. All rights reserved.

\section{Introduction}

Acetylcholine (Ach) is a major excitatory neurotransmitter implicated in many neurological disorders such as Alzheimer's disease and Parkinson's disease [1,2]. Its real-time measurement has proven difficult due to its rapid hydrolysis by acetylcholine esterase. Hence the measurement of its precursors and metabolite choline has proven advantageous [3-5]. The primary technique utilised for Ach measurement has been microdialysis, which, despite recent advances such as using segmented flow [6], remains limited by its spatial and temporal resolution. The development of choline biosensors for the indirect measurement of Ach has become a necessary tool for its detection due to the difficulties associated with multi-enzyme approaches required for direct Ach detection.

The utilisation of first generation choline biosensors incorporating choline oxidase has been reported previously [4,5]. These sensors rely on an enzymatic process involving the catalysis of choline oxidation to glycine betaine with betaine aldehyde as an intermediate and molecular oxygen as the primary electron accep-

\footnotetext{
* Corresponding author.

E-mail address: keeley.l.baker@nuim.ie (K.L. Baker).
}

tor [7], resulting in the production of the signal generating $\mathrm{H}_{2} \mathrm{O}_{2}$ as shown in Eq. (1):

$$
\begin{aligned}
& \left(\mathrm{CH}_{3}\right)_{3} \mathrm{~N}^{+}-\left(\mathrm{CH}_{2}\right)_{2}-\mathrm{OH}+2 \mathrm{O}_{2}+\mathrm{H}_{2} \mathrm{O} \stackrel{\mathrm{ChO}_{\mathrm{x}}}{\longrightarrow} \\
& \left(\mathrm{CH}_{3}\right)_{3} \mathrm{~N}^{+}-\mathrm{CH}_{2}-\mathrm{COOH}+2 \mathrm{H}_{2} \mathrm{O}_{2}
\end{aligned}
$$

Biosensor mediated analyte detection offers a number of advantages over classical microdialysis approaches, including their small size and ability to allow real-time detection of an analyte making them an attractive option for neurochemical monitoring in-vivo. This approach however, still suffers from its own limitations. For example, the incorporation of an oxidase enzyme means these sensors are subject to interference from in-situ fluctuations of both the co-substrate (molecular oxygen) and from electroactive species present in the extracellular fluid (ECF) also detected at the high $(+700 \mathrm{mV})$ oxidation overpotential [8].

We have recently reported the in-vivo characterisation of a novel choline biosensor [9] and here we present the detailed development of this biosensor in-vitro, encompassing extensive sensitivity optimisation, oxygen interference and choline selectivity confirmation.

Excellent choline sensitivity is a critical criteria for the physiological monitoring of choline due to its relatively low concentration in the ECF (approximately $6 \mu \mathrm{M}$ ). The effect of oxygen variability on sensor performance was also determined in an attempt to eliminate any potential interference once implanted in a freely moving 
animal. In addition, a permselective membrane was used in order to reduce the interference contribution from the highly abundant and electroactive species Ascorbic Acid (AA). Finally, implantation of the sensor alongside a microdialysis probe validated that the sensor can detect changes in choline concentrations around the active surface when implanted in living tissue.

\section{Materials and methods}

\subsection{Chemicals and solutions}

The $o$-phenylenediamine (o-PD, 1,2-diaminobenzene, $\geq 98 \%$ ), styrene (Sty, 99\%), methyl methacrylate (MMA, 99\%), cellulose acetate (Mn $\sim 50,000 \mathrm{~g} / \mathrm{mol}$ ), Nafion ${ }^{\circledR}$ ( $5 \mathrm{wt} \%$ in lower aliphatic alcohols in $\mathrm{H}_{2} \mathrm{O}$ ), choline oxidase (ChOx; from Alcaligenes sp., EC 232-840-0, $1 \mathrm{KU}$ ), bovine serum albumin (BSA, fraction $\mathrm{V}$ from bovine plasma), glutaraldehyde (Grade $1,25 \%$ ), polyethyleneimine (PEI, 80\% ethoxylated), $\mathrm{NaCl}$ (SigmaUltra), $\mathrm{NaH}_{2} \mathrm{PO}_{4}$ (Sigma, A.C.S. reagent), $\mathrm{NaOH}$ (SigmaUltra), choline chloride ( $\geq 97 \%$ ), were obtained from Sigma-Aldrich Ireland Ltd (Dublin). In-vitro electrochemical experiments were conducted in a phosphate buffered saline (PBS) solution, $\mathrm{pH} 7.4 ; \mathrm{NaH}_{2} \mathrm{PO}_{4}(40 \mathrm{mM}), \mathrm{NaOH}(40 \mathrm{mM})$, $\mathrm{NaCl}(150 \mathrm{mM})$. Choline chloride (0.1 M), o-PD monomer $(300 \mathrm{mM}$ in $\mathrm{N}_{2}$-saturated PBS), BSA (0.1-1\%), glutaraldehyde (0.1-1\%) and PEI (1-2\%) were always prepared fresh.

\subsection{Working electrode preparation}

5T Teflon ${ }^{\circledR}$-coated Pt/Ir (90\%/10\%) disc electrodes or cylinder electrodes ( $125-\mu \mathrm{m}$ bare diameter, $175-\mu \mathrm{m}$ coated diameter, Advent Research Materials, Suffolk, UK) of aprx. $6 \mathrm{~cm}$ in length were utilised in this study. $3 \mathrm{~mm}$ of Teflon ${ }^{\circledR}$ insulation was stripped from the wire and soldered onto a gold clip (Fine Science Tools $\mathrm{GmbH}$, Heidelberg, Germany) to facilitate an electrical connection to the potentiostat while the opposite end of the wire was freshly cut to form an active surface disc for the biosensor. All cylinder electrodes had an additional $1.0 \pm 0.1 \mathrm{~mm}$ of Teflon stripped away (Fig. 1a). Poly(o-phenylendiamine) (PPD) was electrochemically grown onto the active surface and stored at $4{ }^{\circ} \mathrm{C}$ for a minimum of $3 \mathrm{~h}$ before biosensor constituent addition. The active surface of each electrode was coated with biosensor constituents using a dip absorption method. Briefly, electrodes were dipped for $0.5 \mathrm{~s}$ into monomeric immobilisers MMA or Styrene, followed by incorporation of Nafion ${ }^{\circledR}(5 \%)$ or cellulose acetate (2\%) and then consecutively dipped into ChOx (50 or $500 \mathrm{U} / \mathrm{ml}$ ), BSA (0.1-1\%), glutaraldehyde $(0.1-1 \%)$ and PEI (1-2\%) for a total of 10 layers. 5 min drying incubations were conducted between each layer (Fig. 1b). Sensors were prepared in batches of four, typically taking three days to manufacture. Prior to biosensor utilisation, the sensors were kept at room temperature for a minimum of one hour or stored at $4{ }^{\circ} \mathrm{C}$ until calibrated. Oxygen measurements were conducted using a freshly cut $5 T$ Teflon $^{\circledR}$-coated Pt/Ir (90\%/10\%) disc electrode (125- $\mu \mathrm{m}$ bare diameter, $175-\mu \mathrm{m}$ coated diameter, Advent Research Materials, Suffolk, UK) of aprx. $6 \mathrm{~cm}$ in length. Teflon ${ }^{\circledR}$ insulation was stripped from one end of the wire and soldered into a gold clip (Fine Science Tools GmbH, Heidelberg, Germany).

\subsection{Instrumentation}

A standard three-electrode glass electrochemical cell was used for all sensor calibrations (choline: 0-3 mM and oxygen 0-240 $\mu \mathrm{M}$ ) in $20 \mathrm{~mL}$ PBS. The reference electrode was a saturated calomel electrode (SCE), while bare Pt wire was utilised as the auxiliary electrode. Constant potential amperometry (CPA; $+700 \mathrm{mV}$ (choline), $-650 \mathrm{mV}\left(\mathrm{O}_{2}\right)$ ) performed in all electrochemical experiments were conducted using a custom designed low-noise potentiostat, Biostat IV, ACM Instruments, Cumbria, UK; with a notebook PC (in-vitro) or Mac ${ }^{\circledR}$ a PowerLab interface system (ADInstruments Ltd., Oxford, UK) and LabChart ${ }^{\circledR}$ for Windows and Mac ${ }^{\circledR}$ (Version 6, ADInstruments Ltd).

\subsection{Data analysis}

Data presented as mean \pm standard error (SEM) while $n$ refers to the number of sensors used in each study. Data analysis was conducted with the commercial package of Prism (version 6.04; GraphPad Software, Inc., CA, USA). Unpaired $t$-tests (two-tailed) were also calculated where appropriate using Prism. $P<0.05$ values were deemed statistically significant. Michaelis-Menten non-linear regression analysis (Eq. (2)) was used to provide enzyme kinetic parameters $\mathrm{V}_{\mathrm{MAX}}, \mathrm{K}_{\mathrm{M}}$, Linear Region Slope (LRS) and $\mathrm{K}_{\mathrm{M}} \mathrm{O}_{2}$. $\mathrm{V}_{\mathrm{MAX}}$ is the maximum current value at enzyme-substrate saturation. The Michaelis constant $\mathrm{K}_{\mathrm{M}}$ is the concentration of substrate that gives half of the $V_{\text {MAX }}$ response. LRS was obtained from linear regression analysis which is valid up to half the $\mathrm{K}_{M}$ value. $\mathrm{K}_{\mathrm{M}} \mathrm{O}_{2}$ is the concentration of $\mathrm{O}_{2}$ that gives half the $\mathrm{V}_{\mathrm{MAX}}$ response to choline. The CAD programme SolidWorks and online CAD programme Tinkercad were used for schematic design.

$v=\frac{V_{\max }[S]_{0}}{[S]_{0}+K_{M}}$

\subsection{Sensor calibrations}

The working electrodes were calibrated at room temperature. They were allowed time to settle under the influence of the applied potential until the non-faradaic current had reached a stable baseline. When this was achieved, calibrations were performed by addition of aliquots of analyte into the buffer solution. For all calibrations additions were made every $4 \mathrm{~min}$ into the buffer which was stirred briefly (20s) after the addition of each aliquot. The current response was taken immediately before the next injection.

\subsection{Monitoring dissolved oxygen}

Biosensor and oxygen sensor $(-650 \mathrm{mV}$ vs SCE) data were recorded simultaneously. The PBS was de-aerated by vigorously purging the solution with $\mathrm{N}_{2}$ (BOC Ireland, average $\mathrm{O}_{2}$ content $2 \mathrm{ppm}$, maximum content $5 \mathrm{ppm}$ ) for at least $30 \mathrm{~min}$ before recording began [10]. A $\mathrm{N}_{2}$ atmosphere was maintained over the cell during biosensor and oxygen sensor settling. Aliquots of analyte were introduced into the buffer and stirred briefly. The $\mathrm{N}_{2}$ source was removed to facilitate the natural air equilibration of the solution where the $\mathrm{O}_{2}$ concentration was taken as $100 \%$ at air saturation $(200 \mu \mathrm{M})$.

Some experiments were carried out under continuous forced convection by the introduction of either air (from a RENA air pump) or $\mathrm{N}_{2}$ into the buffer. Aliquots of analyte were introduced during $\mathrm{N}_{2}$ saturation and monitored during the transition to air saturation. The use of either natural air equilibration, or forced convection is clearly indicated in the text.

\subsection{Surgical procedures}

As described in our previous work [9], anesthesia was achieved in 200-300 g male wistar rats (Charles River Laboratories International, Inc., UK) using the volatile anaesthetic isoflurane (4\% in air) for induction and maintenance (1.5-3.0\%; IsoFlo ${ }^{\circledR}$, Abbott, UK) using a Univentor 400 Anaesthesia Unit (AgnTho's AB, Sweden). Anaesthetised animals were anchored into a stereotaxic frame and the sensors implanted following a previously described procedure 

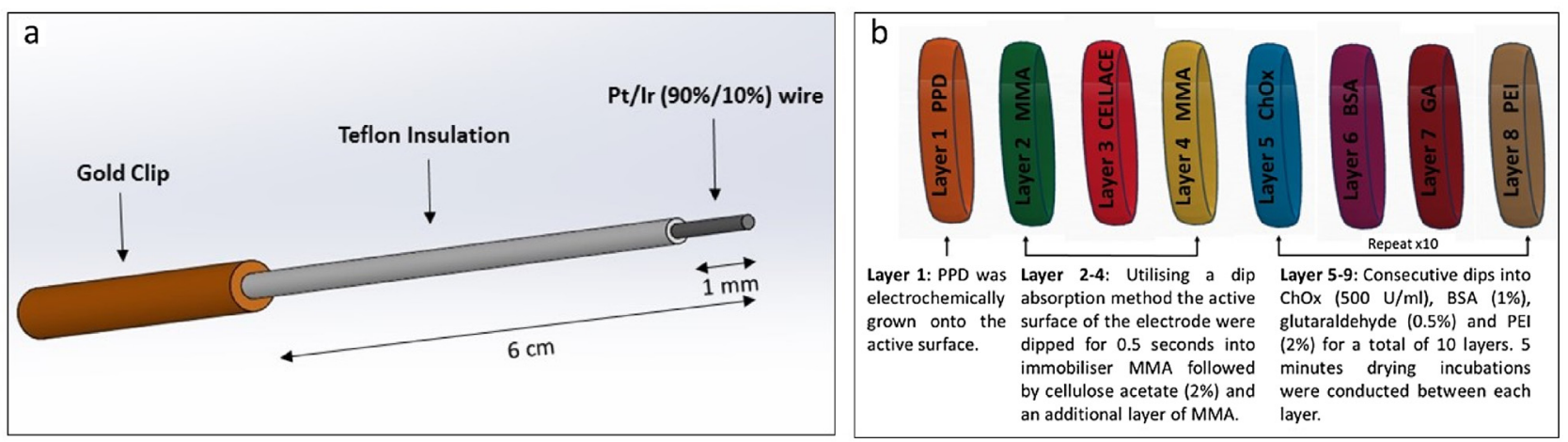

Fig. 1. a. Schematic of an electrode. b. Schematic of the dip coat layering modification made to an electrode for choline biosensor construction.

[9,11]. The skull was levelled between bregma and lambda, and the striatum was located using the following coordinates: $\mathrm{A} / \mathrm{P}+1.0$, $\mathrm{M} / \mathrm{L} \pm 2.5$ from bregma, and $\mathrm{D} / \mathrm{V}-6.0$ from dura. Sensors were co-implanted with a microdialysis probe. The in-vivo reference electrode utilised was a $200-\mu \mathrm{m}$ bare diameter $8 \mathrm{~T} \mathrm{Ag}$ wire (Advent Research Materials) implanted in the cortex while the 8T Ag wire auxillary electrode was fixed to one of the four support screws (Fine Science Tools $\mathrm{GmbH}$ ) on the skull and secured using dental acrylate (Dentalon ${ }^{\circledR}$ Plus, Heraeus-Kulzer, Germany). Microdialysis was conducted using MBR-4 (BASi, West Lafayette, IN, USA) probes. Saline $(0.9 \%)$ and analgesia (Buprecare ${ }^{\circledR}$, AnimalCare Ltd., UK) was administered to animals. Prior to being connected to the potentiostat, animals were allowed to recuperate from the surgery for $24 \mathrm{~h}$. All work was carried out under license in accordance with the European Communities Regulations 2002 (Irish Statutory Instrument 566/2002 - Amendment of Cruelty to Animals Act 1876).

\subsection{Experimental conditions in-vivo}

Animals were singly house and maintained in Raturn ${ }^{\circledR}$ sampling cage systems (BASi, West Lafayette, IN, USA) with ad libitum access to food. The animals were housed in a facility with a $12 \mathrm{~h}$ light/dark cycle. Sensors connected directly to the potentiostat via a six-pin Teflon $^{\circledR}$ socket (MS363, Plastics One, Roanoke, VA, USA) using a flexible screened six core cable (363-363 6TCM, Plastics One), thus allowing the animals to move freely with simultaneous and continuous connection to the potentiostat while in their home bowl. To allow background currents from the electrodes to stabilise, each animal was allowed $24 \mathrm{~h}$ rest after the appropriate applied potential was initially applied. Furthermore, to eliminate background noise from AC, a low-pass digital filter cut-off $(50 \mathrm{~Hz})$ was applied and all data was recorded at $40 \mathrm{~Hz}$. To switch between solutions for perfusions in microdialysis probes, a UniSwitch syringe selector (BASi) was utilised.

\section{Results and discussion}

\subsection{Kinetic characteristics of the basic choline biosensor designs}

Both first and second generation biosensors have been developed for choline monitoring. These biosensors have utilised various transducers and immobilisation methods. The incorporation of ChOx has proved advantageous in choline sensor development and its immobilisation has been achieved through various methods including drop casting or electrostatic interaction of the enzyme following immersion into the enzyme solution $[3,12,4,13]$. Synthetic polymers have also been used for the entrapment of enzymes [14], an approach successfully demonstrated previously using monomeric styrene [15]; a liquid at room temperature. Therefore,
Table 1

Comparison of $\mathrm{V}_{\mathrm{MAX}}, \mathrm{K}_{\mathrm{M}}$ and sensitivities (LRS) of the choline biosensor designs using $50 \mathrm{U} / \mathrm{mL}$ ChOx.

\begin{tabular}{|c|c|c|c|c|}
\hline & Design $(n=4)$ & $\mathrm{V}_{\mathrm{MAX}}(\mathrm{pA})$ & $\mathrm{K}_{\mathrm{M}}(\mu \mathrm{M})$ & $\operatorname{LRS}(\mathrm{pA} / \mu \mathrm{M})$ \\
\hline 1 & Sty-(ChOx $)_{1}$ & $4.3 \pm 3.2$ & $81.1 \pm 41$ & $0.002 \pm 0.01$ \\
\hline 2 & Sty- $(\mathrm{ChOx})^{30 \mathrm{~min}}$ & $234 \pm 30$ & $555 \pm 171$ & $0.16 \pm 0.1$ \\
\hline 3 & Sty-(ChOx $)_{10}$ & $34.2 \pm 11.9$ & $308 \pm 337$ & $0.44 \pm 0.1$ \\
\hline 4 & Sty- $(\mathrm{ChOx})_{10}-\mathrm{GA}$ & $120 \pm 33$ & $357 \pm 461.6$ & $0.21 \pm 0.2$ \\
\hline 5 & Sty-(ChOx:BSA $)_{10}-\mathrm{GA}$ & $2184 \pm 95$ & $1966 \pm 215$ & $1.92 \pm 0.1$ \\
\hline 6 & Sty-(ChOx:BSA-PEI $)_{10}-$ GA & $3880 \pm 420$ & $507 \pm 138$ & $10.0 \pm 0.1$ \\
\hline
\end{tabular}

Subscript numbers indicate number of $1 \mathrm{sec}$ ChOx applications. Superscript numbers indicate duration of a single $\mathrm{ChOx}$ application.

this represents a simple method for the entrapment of choline oxidase using the dip coating approach to enzyme loading [16] and was used as the initial approach for incorporating ChOx onto the transducer.

Designs 1-3 successfully incorporated ChOx into the styrene monomer immobilised on a Pt disc $(175 \mu \mathrm{m})$ (Table 1$)$. The sensitivity of these designs, albeit low, demonstrated that $\mathrm{ChOx}(50 \mathrm{U} / \mathrm{mL})$ was immobilised onto the electrode surface and can be used for the detection of choline. The sensitivity of Design $3(0.44 \pm 0.1 \mathrm{pA} / \mu \mathrm{M})$ was significantly higher than designs $1(0.002 \pm 0.01 \mathrm{pA} / \mu \mathrm{M}$, $p=0.002)$ and $2(0.16 \pm 0.1 \mathrm{pA} / \mu \mathrm{M}, p=0.0029)$ and was used as the basis for further modifications (Fig. 2a).

Design 4 incorporated Glutaraldehyde (GA), an effective crosslinking agent that has been used frequently in biosensor design $[17,18]$. Here, the inclusion of GA alone did not improve sensitivity $(0.21 \pm 0.2 \mathrm{pA} / \mu \mathrm{M})$. When used in conjunction with Bovine Serum Albumin (BSA), a lysine rich protein which provides protection to the underlying ChOx, sensitivity significantly increased $(1.92 \pm 0.1 \mathrm{pA} / \mu \mathrm{M}, p=0.0048)$. This is achieved as GA preferentially binds to the BSA, liberating ChOx active sites for diffusion of choline and substrate turnover. The polycation polyethyleneimine (PEI) has been previously used to enhance enzyme immobilisation [19-21] and stabilisation [22] in biosensor design. In addition, inclusion of PEI into biosensor design has previously dramatically increased sensor sensitivity $[23,21]$, reduced the Michaelis constant $K_{M}$ and increased the linear region slope [24]. This is attributed to the formation of polyanionic/polycationic complexes and by decreasing the electrostatic repulsion between the enzyme substrate and biosensor components [24]. The inclusion of PEI in Design 6 significantly $(p=0.0021)$ increased the sensitivity $(10.0 \pm 0.1, \mathrm{n}=4)$ and reduced the $K_{M}$ (Design 6 vs. 5; Fig. 2a and b).

\subsection{Optimisation of choline oxidase immobilisation}

The previous section demonstrates that ChOx can be successfully immobilised onto a Pt disc electrode using monomeric styrene and dip absorption. However, due to the low concentration of 
a

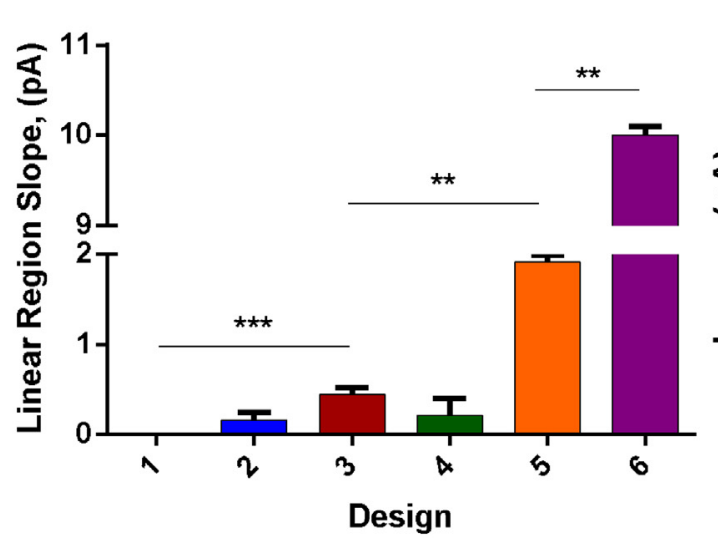

b

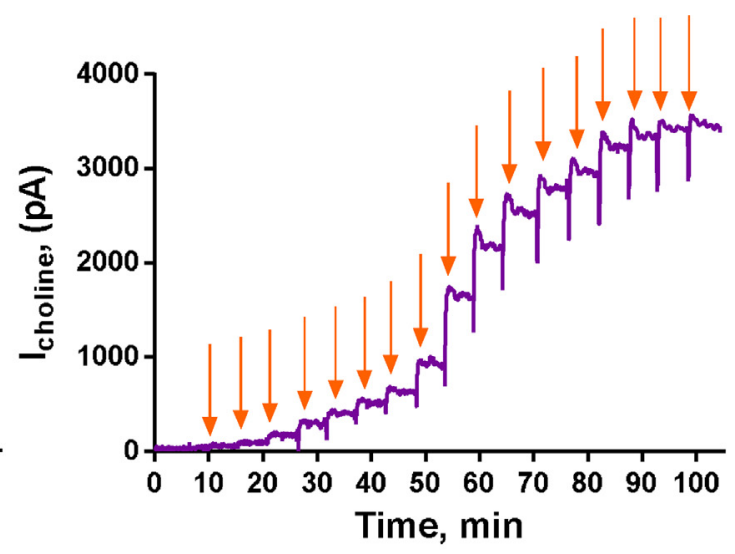

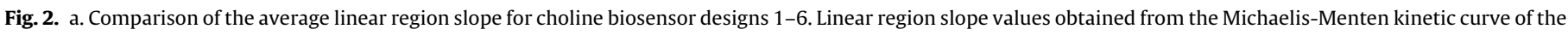

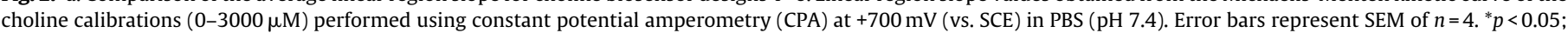

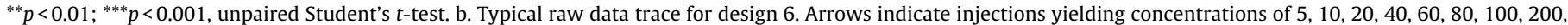
$400,600,800,1000,1500,2000,2500$ and $3000 \mu \mathrm{M}$ of choline.

Table 2

Comparison of $\mathrm{V}_{\mathrm{MAX}}, \mathrm{K}_{\mathrm{M}}$ and sensitivities of the choline biosensor designs using $500 \mathrm{U} / \mathrm{mL}$ ChOx.

\begin{tabular}{|c|c|c|c|c|}
\hline & Design & $\mathrm{V}_{\mathrm{MAX}}(\mathrm{nA})$ & $\mathrm{K}_{\mathrm{M}}(\mu \mathrm{M})$ & $\operatorname{LRS}(\mathrm{pA} / \mu \mathrm{M})$ \\
\hline 7 & Sty- $(\mathrm{ChOx})_{10}-\mathrm{GA} n=4$ & $19.2 \pm 3.7$ & $621.5 \pm 290.8$ & $22.5 \pm 0.3$ \\
\hline 8 & Sty-(ChOx)(GA) $n=4$ & $7.8 \pm 0.5$ & $188.8 \pm 36.0$ & $19 \pm 1$ \\
\hline 9 & Sty-(ChOx)(PEI)(GA) $n=3$ & $6.8 \pm 0.3$ & $283.4 \pm 33.1$ & $15 \pm 1$ \\
\hline 10 & Sty-(ChOx)(GA)(PEI) $n=3$ & $6.7 \pm 0.3$ & $367.7 \pm 41.4$ & $12 \pm 0.3$ \\
\hline 11 & Sty-(ChOx)(BSA)(GA) $n=3$ & $8.5 \pm 0.6$ & $899.8 \pm 154.8$ & $8.6 \pm 0.2$ \\
\hline 12 & Sty-(ChOx)(BSA)(GA)(PEI 1\%) $n=4$ & $9.5 \pm 0.2$ & $249.0 \pm 12.9$ & $22 \pm 1$ \\
\hline 13 & Sty-(ChOx)(BSA)(GA)(PEI 2\%) $n=3$ & $6.9 \pm 0.2$ & $237.7 \pm 15.2$ & $17 \pm 1$ \\
\hline 14 & Sty-(ChOx)(BSA)(GA0.5\%)(PEI) $n=4$ & $11.4 \pm 0.1$ & $298.6 \pm 8.6$ & $20 \pm 1$ \\
\hline 15 & Sty-(ChOx)(BSA)(GA0.5\%)(PEI2\%) $n=3$ & $12.7 \pm 0.7$ & $269.1 \pm 38.5$ & $27 \pm 1$ \\
\hline 16 & MMA-(ChOx)(BSA)(GA0.5\%)(PEI2\%) $n=4$ & $15.6 \pm 0.4$ & $302.3 \pm 24.1$ & $30 \pm 1$ \\
\hline
\end{tabular}

choline in the brain (ca. $6 \mu \mathrm{M})$ it was essential that the sensor sensitivity was increased further. Table 2 shows the sensitivity of electrode designs 7-15 which utilised an increase in enzyme activity from 50 units $/ \mathrm{mL}$ (Table 1 ) to 500 units $/ \mathrm{mL}$. A comparison between designs 4 and 7 demonstrates a significant $(p=0.0030)$ increase in sensitivity from $0.21 \pm 0.2 \mathrm{pA} / \mu \mathrm{M}$ to $22.5 \pm 0.3 \mathrm{pA} / \mu \mathrm{M}$. The incorporation of GA onto each layer (design 8) successfully reduced the $K_{M}(621.5 \pm 290.8$ to $188.8 \pm 36.0)$ while having no significant $(p=0.8240)$ effect on the overall sensitivity of the sensor. In contrast to the positive effect of BSA in design 5 , the inclusion of BSA in design 11 yielded higher BSA deposition in a single layer when applied after ChOx immobilisation and was detrimental to sensitivity, yet, when used in conjunction with PEI (Design $12)$, sensitivity was increased dramatically $(22 \pm 1 \mathrm{pA} / \mu \mathrm{M})$ when compared to PEI alone (Design $10 ; 12 \pm 0.3 \mathrm{pA} / \mu \mathrm{M}$ ). This demonstrates the protective nature of the BSA application when adding additional constituents into the design that can hinder the free diffusion of the substrate to the active site of the enzyme. Designs 12 to 15 demonstrate the effect of GA and PEI concentration on sensitivity. The highest sensitivity was achieved using $0.5 \%$ GA and 2\% PEI. Additionally, this design was utilised in conjunction with the monomer methylmethacrylate (MMA). The polymerised form of MMA has been used in the development of sensors previously reported $[25,26]$. Similar to styrene, MMA is a monomeric liquid at room temperature and thus ideal for use with this type of immobilisation. The sensitivity of this design was increased slightly, though it was not statistically different $(p=0.5073)$ to that of styrene (Design 15). As, the $\mathrm{K}_{\mathrm{M}}$ and $\mathrm{V}_{\mathrm{MAX}}$ were also increased, this design (16) was chosen for all further experiments.

\section{3. $\mathrm{O}_{2}$ dependence studies}

A limitation of first generation biosensors is that changes in $\mathrm{O}_{2}$ concentration may cause interference in the substrate response [27]. Therefore, one criteria to which a biosensor must adhere is a low sensitivity to changes in oxygen over the range of substrate and oxygen concentrations relevant to the intended model [28]. As the choline biosensor is intended for in-vivo monitoring, where normal $\mathrm{O}_{2}$ concentrations in the brain have been reported between 40 and $80 \mu \mathrm{M}$, the need for oxygen interference studies to characterise biosensor functionality is paramount [29-31]. One approach to overcome this has been the development of 'second generation biosensors' which replaces oxygen with an artificial mediator [32]. The development of a choline biosensor using a different type of mediated system by Garguilo et al. has been reported which incorporates the immobilisation of choline oxidase and horseradish peroxidase using a cross-linkable redox polymer [33] which mediates electron transfer between the peroxidase and the electrode [34]. Although the use of mediators may aid in addressing the oxygen dependence of biosensors, such devices can suffer from leaching of the mediator from the enzyme layer [29] causing toxicity problems [35]. In addition, the reported insensitivity to oxygen interference has been questioned for certain mediators [36].

An alternative to the second generation biosensor is the use of fluorochemical pasting liquids, with high $\mathrm{O}_{2}$ solubility. These have mainly been used with carbon-based electrodes. For example, studies have highlighted the use of a variety of fluorocarbon oils (for example Kel-F and Nafion ${ }^{\circledR}$ ) for their internal oxygen reservoir and supply properties in carbon paste glucose biosensors [37-39]. 
a

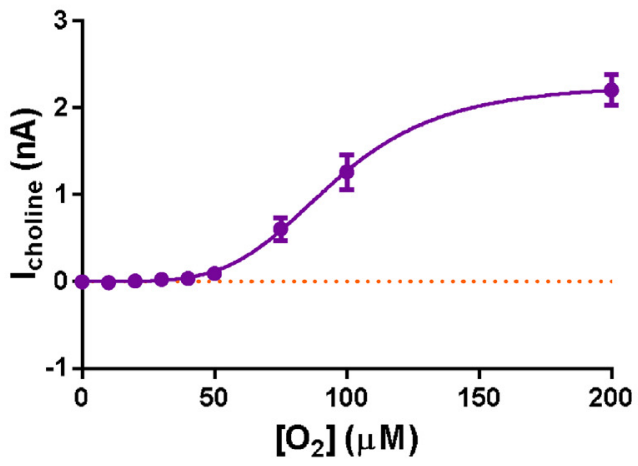

C

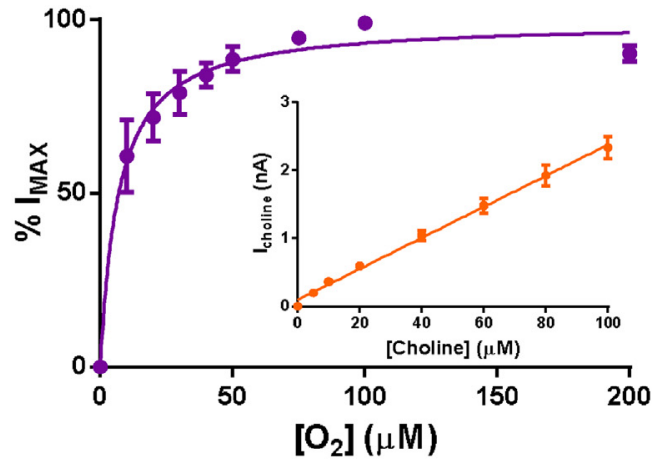

b

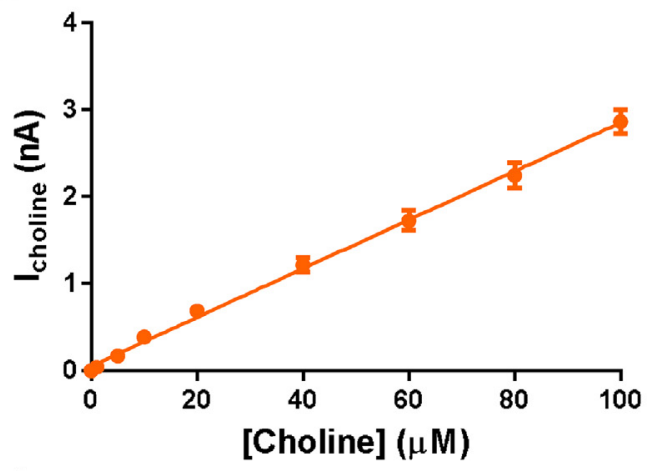

d

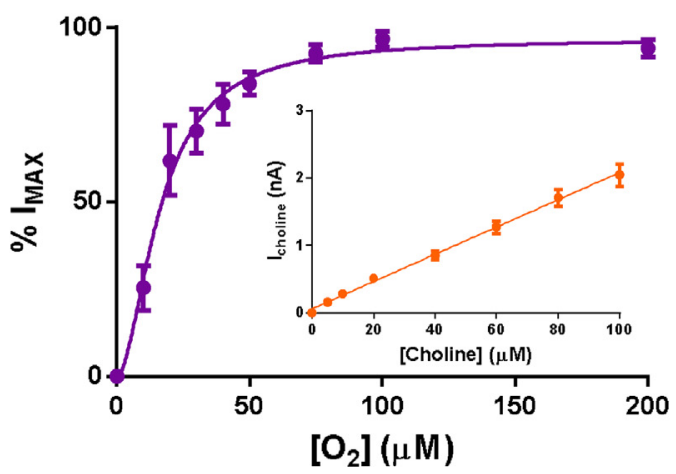

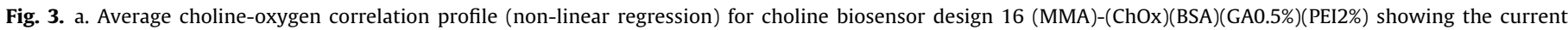

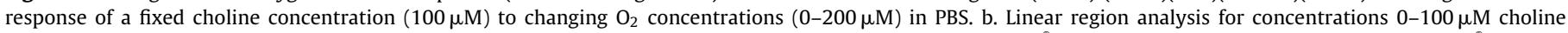

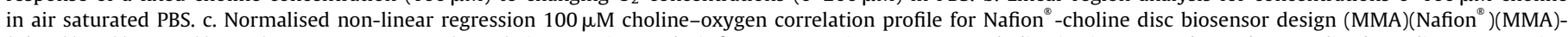

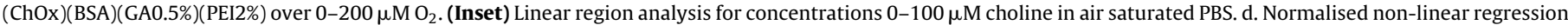

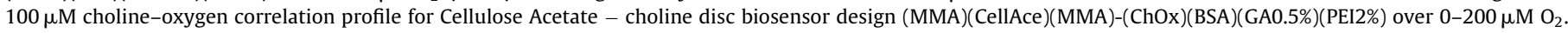

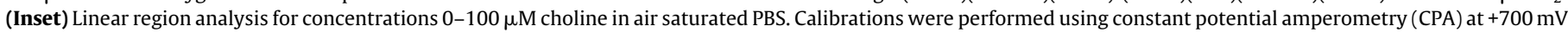
(vs. SCE) in PBS (pH 7.4). Error bars represent SEM of $n=4$.

Choline biosensor design 16 displays a high degree of oxygen dependence and achieved only $3 \%$ of the Imax current at $50 \mu \mathrm{M} \mathrm{O} \mathrm{O}_{2}$ (Fig. 3a) while just retaining superior analyte sensitivity at atmospheric oxygen concentrations $(200 \mu \mathrm{M})$ (Fig. 3b). Therefore, sensor design 16 was considered unsuitable for in-vivo detection of choline. This design was then modified to include the use of Nafion ${ }^{\circledR}$, which has previously been reported to act as an $\mathrm{O}_{2}$ reservoir ((MMA)(Nafion $\left.{ }^{\circledR}\right)$ (MMA)(ChOx)(BSA)(GA0.5\%)(PEI2\%)) [39,37]. Indeed, diffusion of gases such as $\mathrm{O}_{2}$ through Nafion ${ }^{\circledR}$ have been well characterised in the fuel cell literature where the driving forces for permeation include hydration level, pressure and temperature $[40,41]$. For Nafion ${ }^{\circledR}$ it is recognised that permeation is of a solely diffusive nature [41]. Fig. $3 \mathrm{c}$ shows a choline- $\mathrm{O}_{2}$ correlation plot for a fixed concentration of choline $(100 \mu \mathrm{M})$ over a $0-200 \mu \mathrm{M} \mathrm{O}_{2}$ concentration range. The effect of oxygen on the choline current is presented as normalised current using the air-saturated choline current as $100 \%$. A comparison of the $\mathrm{K}_{\mathrm{M}}\left(\mathrm{O}_{2}\right)$ value of this design $(6.9 \mu \mathrm{M}$, Fig. $3 \mathrm{c})$ with the original MMA design $16(89.8 \mu \mathrm{M})$ illustrates the positive effect of Nafion ${ }^{\circledR}$ on reducing the $\mathrm{O}_{2}$ dependence of the sensor. This significant decrease in the $\mathrm{K}_{\mathrm{M}}\left(\mathrm{O}_{2}\right)$ obtained by introducing Nafion ${ }^{\circledR}$ led us to investigate if it was indeed acting as an $\mathrm{O}_{2}$ reservoir, or simply a diffusional barrier. Cellulose Acetate (CellAce), which doesn't have the ability to act as an oxygen reservoir, was introduced into the biosensor design to test if the additional layer had an effect on the $\mathrm{O}_{2}$ dependence of the sensor ((MMA)(CellAce)(MMA)(ChOx)(BSA)(GA0.5\%)(PEI2\%)). The use of CellAce in the design produced a similar significant decrease in the $\mathrm{K}_{\mathrm{M}}\left(\mathrm{O}_{2}\right)$ concentration to $16.5 \mu \mathrm{M}$ (Fig. 3d). The effect of Nafion ${ }^{\circledR}$ or cellulose acetate modification on the sensor design does not alter the biosensor's sensitivity to choline (Fig. 3c and 3d insets). The inclusion of Nafion $^{\circledR}$ resulted in a sensitivity of $22 \pm 1 \mathrm{pA} / \mu \mathrm{M}, n=4$, similar to the design including cellulose acetate $(20 \pm 1 \mathrm{pA} / \mu \mathrm{M}, n=4)$. This was also similar to the previous MMA design (16) with a sensitivity of $30 \pm 1 \mathrm{pA} / \mu \mathrm{M}, n=4$ (Table 2 ). Interestingly, the improvement achieved by the inclusion of both Nafion ${ }^{\circledR}$, the oxygen carrying fluorocarbon, or the cellulose acetate used as a diffusional barrier, yielded similar $\operatorname{Km}\left(\mathrm{O}_{2}\right)$ values suggesting that Nafion ${ }^{\circledR}$ is not functioning as an internal oxygen supply in this case.

\subsection{Comparison of disk and cylinder geometries}

In the previous section, the effect of altering the diffusion parameters of the sensor using additional layering with cellulose acetate showed a dramatic improvement in the level of $\mathrm{O}_{2}$ dependence. In order to investigate if this could be improved upon further, the geometry of the cellulose acetate modified choline biosensor was changed from a disc electrode which is subject to radial diffusion, to a cylinder electrode which is subject to both radial and linear diffusion. When considering the enzyme loading achieved by layering on the sensor surface, this increased surface area will also change the distribution of the enzyme around the cylinder surface. This approach has previously been shown to decrease $\mathrm{O}_{2}$ dependence in other biosensors [28]. The current values at $\mathrm{I}_{100} \mu \mathrm{M}$ increased ( $p=0.0042$ ) from $2.27 \pm 0.04 \mathrm{nA}, n=3$ on a disc electrode to $61.19 \pm 10.06 \mathrm{nA}, n=4$ on a cylinder electrode by increasing the surface area from $24 \mu \mathrm{m}^{2}$ to $598 \mu \mathrm{m}^{2}$, respectively (Fig. 4a). This represents a reduction in current density from $184 \pm 3 \mathrm{nA} / \mathrm{mm}^{2}$, 
a

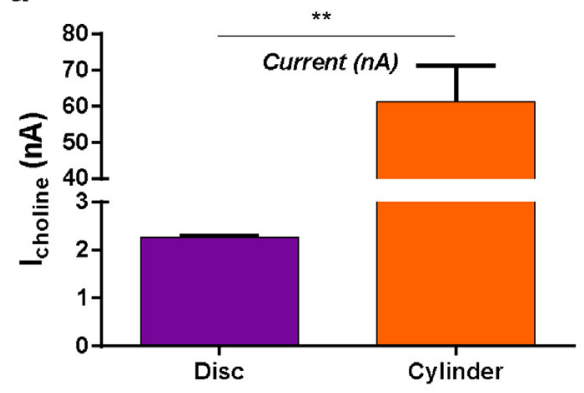

C

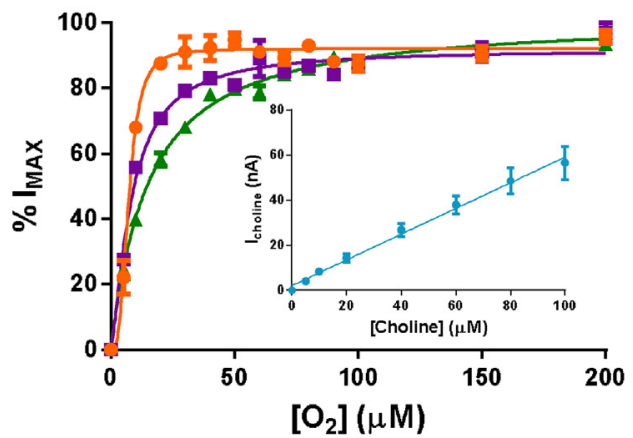

b

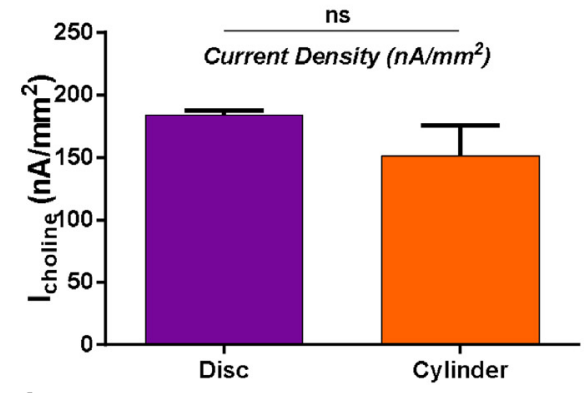

d

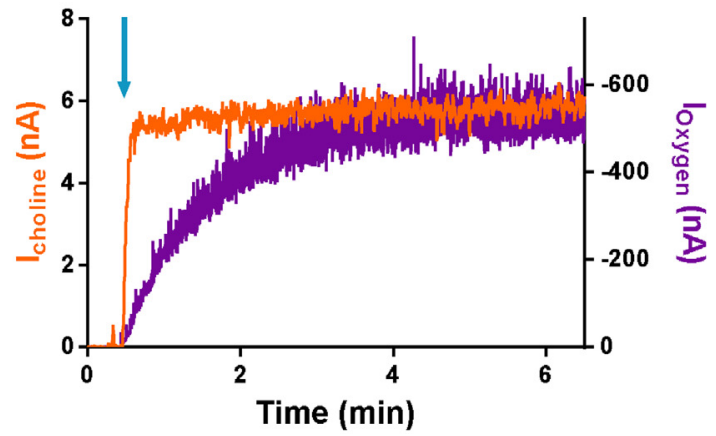

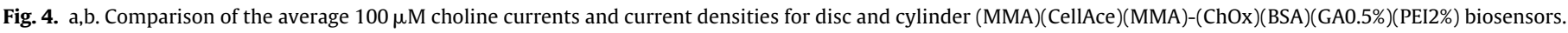

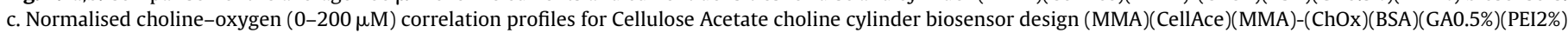

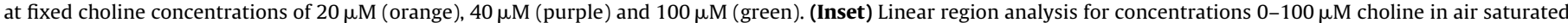

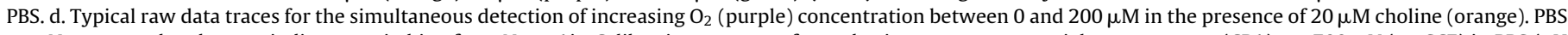

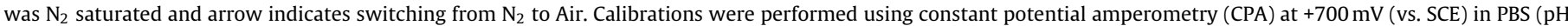

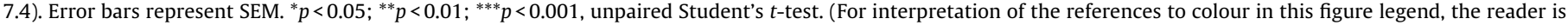
referred to the web version of this article.)

$n=3$ on a disc to $151 \pm 25 \mathrm{nA} / \mathrm{mm}^{2}, n=3$ on the cylinder (Fig. $4 \mathrm{~b}$ ). This reduction in current density occurs despite an overall increase in sensitivity indicating that we have decreased the enzyme loading at the sensor surface thus affecting the diffusion of the substrate choline. This would not be possible on the disc electrode as the current values are too low. High levels of substrate detection require increased concentration of $\mathrm{O}_{2}$; an effect resulting in high $\mathrm{O}_{2}$ interference at high substrate concentrations. Fig. 4c illustrates that the $\mathrm{O}_{2}$ dependence of the cylinder electrode design is comparable with that of the disc electrode shown in Fig. 3d. A comparable $\mathrm{K}_{\mathrm{M}}\left(\mathrm{O}_{2}\right)$ of $15.02 \mu \mathrm{M}$ was achieved for the cylinder electrode at $100 \mu \mathrm{M}$ choline, a slight decrease from the $\mathrm{K}_{\mathrm{M}}\left(\mathrm{O}_{2}\right)$ of $16.5 \mu \mathrm{M}$ obtained using a disc geometry. This suggests that the increase in sensitivity of the cylinder design by increasing the surface area has not affected the level of $\mathrm{O}_{2}$ dependence. In addition to the sensitivity of the sensor, increasing the substrate concentration has previously been shown to have a negative effect on the $\mathrm{O}_{2}$ dependence of a glucose biosensor [27], although, this has not been the case for previous choline biosensors. Burmeister et al. reported minimal $\mathrm{O}_{2}$ dependence of approximately $15 \%$ at a microelectrode array between $50 \mu \mathrm{M}$ and $200 \mu \mathrm{M} \mathrm{O}_{2}$. This percentage variation did not change over a choline concentration range of $25-100 \mu \mathrm{M}$ choline displaying similar levels of oxygen dependence regardless of choline concentration.

Fig. 4c illustrates the relationship between increasing choline concentration $(20,40$ and $100 \mu \mathrm{M})$ and $\mathrm{O}_{2}$ dependence of the (MMA)(CellAce)(MMA)(ChOx)(BSA)(GA0.5\%)(PEI2\%) biosensor. The increase in substrate concentration shows a marginal increase in the $\mathrm{O}_{2}$ dependence of the biosensor; increasing the $\mathrm{K}_{\mathrm{M}}\left(\mathrm{O}_{2}\right)$ from $7.20 \mu \mathrm{M}$ to $8.28 \mu \mathrm{M}$ and $15.0 \mu \mathrm{M}$ at choline concentrations of $20 \mu \mathrm{M}, 40 \mu \mathrm{M}$ and $100 \mu \mathrm{M}$, respectively (Fig. 4c). At
$20 \mu \mathrm{M}$ choline the potential fluctuation in choline current between the $\mathrm{O}_{2}$ concentration range of $30-80 \mu \mathrm{M}$ is approximately $2 \%$. The current value at $50 \mu \mathrm{M} \mathrm{O}_{2}$ is $1 \%$ lower than that achieved in air $(200 \mu \mathrm{M})$. Fig. $4 \mathrm{~d}$ illustrates a typical raw data trace of the simultaneous recording of choline and oxygen in-vitro demonstrating the effective detection of choline at low $\mathrm{O}_{2}$ concentrations. As invivo estimations of choline concentration are as low as $6 \mu \mathrm{M}$ in the brain [42], the ability of the choline biosensor to function free of $\mathrm{O}_{2}$ interference even at high concentrations of choline $(20 \mu \mathrm{M})$, demonstrates the biosensors specificity for choline detection, an important prerequisite when utilising biosensors in freely moving animals which have fluctuating choline and $\mathrm{O}_{2}$ concentrations.

\subsection{Interference studies}

The high endogenous concentrations of electroactive species, principally ascorbic acid (AA), in the extracellular fluid remains an important consideration in the development of any biosensor intended for in-vivo applications [43]. The estimated basal concentration of AA in the brain is approximately $400 \mu \mathrm{M}$ [44], which can readily mask the current response attributed to choline which is approximately $6 \mu \mathrm{M}$ in-vivo. The inclusion of the polymer, poly(o-phenylendiamine) (PPD), for interference rejection has been widely utilised in many biosensors detecting a number of analytes including glucose [45] and glutamate [46]. The advantage of this permselective polymer is its permeability to $\mathrm{H}_{2} \mathrm{O}_{2}$ while simultaneously blocking larger interferent molecules such as the aforementioned AA [15]. The inclusion of PPD into the choline biosensor design significantly $(p<0.0001)$ reduced the current response towards $1000 \mu \mathrm{M}$ AA from $600 \pm 30 \mathrm{nA}, n=4$ (Fig. 5a) to $0.29 \pm 0.12 \mathrm{nA}, n=7$ (Fig. $5 \mathrm{~b}$ ) due to a 'self-blocking' phenomenon whereby at high concentrations of $A A$, the polymer 
a

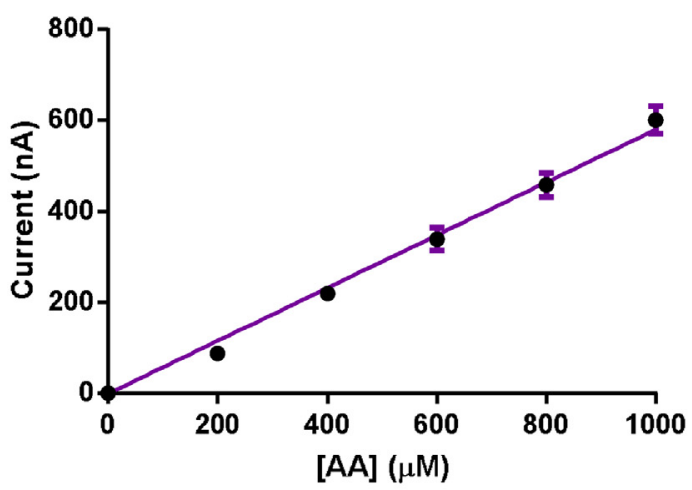

b

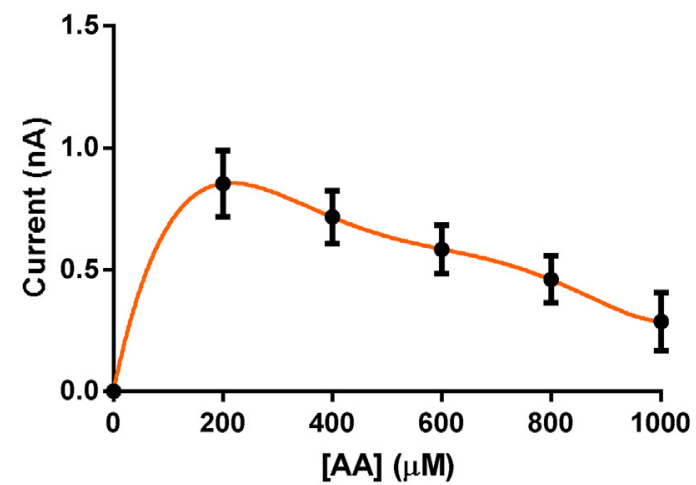

C

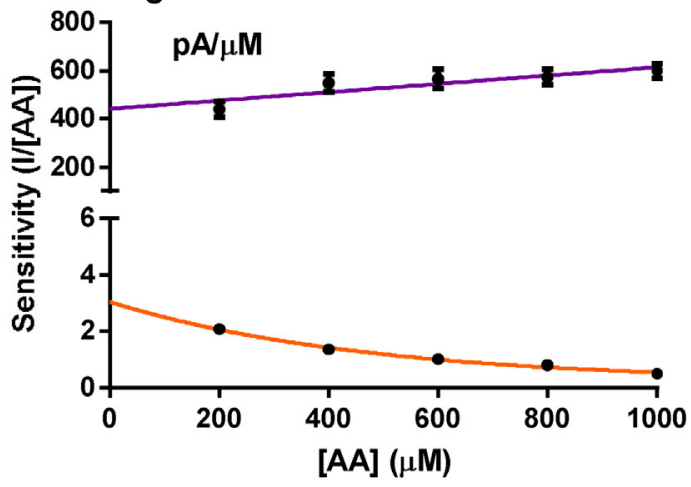

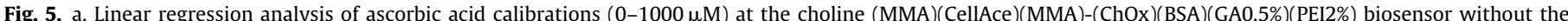

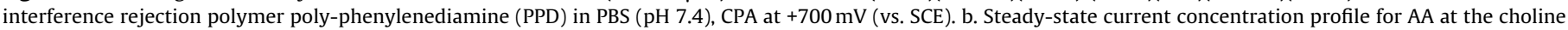

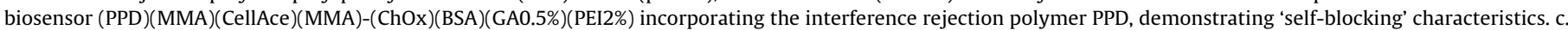

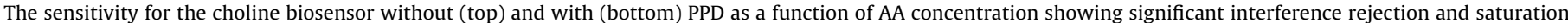
characteristics at physiological levels.

a

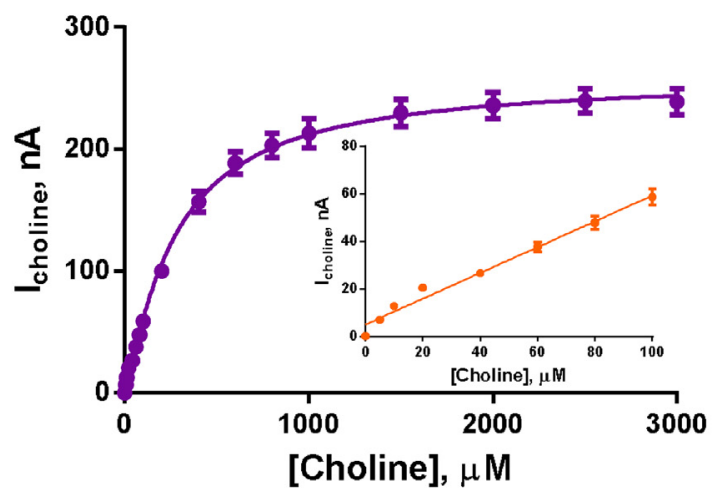

b

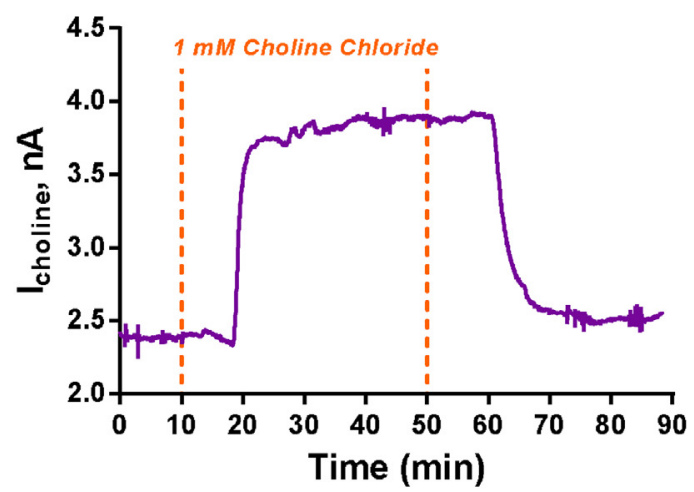

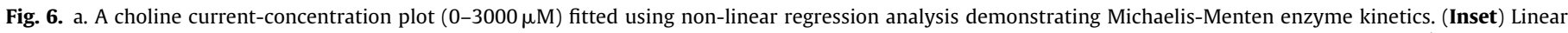

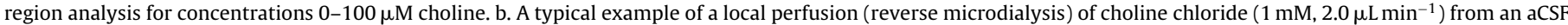

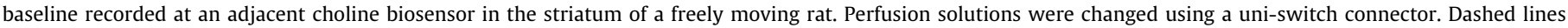
illustrate the point at which the solutions were switched.

becomes increasingly impermeable to AA, gradually decreasing the sensor's sensitivity towards the interferent until reaching a steadystate $[47,48]$. The response to AA is better understood in terms of the sensitivity (I/[AA]) as a function of concentration (Fig. 5c). Without the PPD layer present the calibration is linear and the sensitivity is concentration independent with a values of $600 \pm 30 \mathrm{pA} / \mu \mathrm{M}$. With PPD mass transport inhibition in the polymer results in a significantly reduced sensitivity of $2.1 \pm 0.2 \mathrm{pA} / \mu \mathrm{M}$. The inclusion of PPD does not alter the diffusion of $\mathrm{H}_{2} \mathrm{O}_{2}$ through the polymer or affect the sensitivity of the sensor to choline $540 \pm 30 \mathrm{pA} / \mu \mathrm{M}, n=4$ (with PPD) vs. $560 \pm 30 \mathrm{pA} / \mu \mathrm{M}$ (without PPD).

\subsection{In-vivo choline detection}

We have developed a highly sensitive and selective choline biosensor by rigorously optimising the sensor core constituents (Fig. 2), minimising interference from $\mathrm{O}_{2}$ fluctuations (Fig. 3 and 4), and eliminating current contributions 
for the most relevant endogenous electroactive analyte found in the brain (Fig. 5). The final choline biosensor design of (PPD)(MMA)(CellAce)(MMA)(ChOx)(BSA)(GA0.5\%)(PEI2\%) based on a cylinder electrode geometry gives a current value of $58.6 \pm 3.4 \mathrm{nA}$ at $100 \mu \mathrm{M}$ choline (Fig. 6a).

Preliminary in-vivo characterisation of the choline biosensor signal was obtained by testing the response of the sensor in the striatum of a freely moving rat. The biosensor was co-implanted with a microdialysis probe and an initial perfusion of aCSF decreased the current to a baseline of $2.59 \pm 0.15 \mathrm{nA}$. This increased to $3.99 \pm 0.06 \mathrm{nA}(p=0.0087)$ after $15 \pm 1 \mathrm{~min}$ upon switching from aCSF to $1 \mathrm{mM}$ choline chloride (Fig. $6 \mathrm{~b}$ ). Subsequent perfusion of aCSF resulted in a return to baseline levels $(2.69 \pm 0.10 \mathrm{nA}$, $p=0.3473$ ). This result demonstrates that the biosensor successfully responds to changes in extracellular choline levels when perfused locally.

\section{Conclusions}

The detailed development of a highly sensitive choline biosensor has been presented. The incorporation of biocompatible monomers for the entrapment of the biological recognition element, choline oxidase, provided the basis for choline sensing. The sensitivity of the biosensor was optimised using bovine serum albumin and glutaraldehyde. The specific formulation of the various constituents ultimately yielded excellent sensitivity towards choline indicating potential suitability for further studies in the brain where choline concentrations are in the low $\mu \mathrm{M}$ range.

The level of oxygen dependence of the sensor was examined; an important parameter, key when designing a first generation biosensor for in-vivo monitoring applications. The physiological fluctuation of this enzyme co-factor can lead to inaccurate interpretations of substrate monitoring in-vivo if the sensor is subject to oxygen interference. Incorporation of cellulose acetate, and changing the geometric surface area for choline detection, produced a design where changes in oxygen over physiological concentration ranges had negligible effect on the choline signal. Also, electroactive interference from endogenous ascorbic acid can negate detection of low concentration analytes such as choline, as this species is easily detected at the same potential using amperometry. We have shown that the electropolymerisation of poly(phenylenediamine) as an underlayer on the platinum surface removes ascorbate interference without affecting choline sensitivity.

Finally, the implantation of this optimised $\mathrm{Pt}_{\text {cyl }}$ (PPD)(MMA)(CellAce)(MMA)(ChOx)(BSA)(GA)(PEI) biosensor in the striatum of a freely-moving rat confirmed the sensors ability to detect choline in living tissue; the sensor successfully monitored exogenously administered choline through a co-implanted microdialysis probe. Future in-vitro work will concentrate on other key operational characteristics such as extending the interference characterisation to other appropriate endogenous analytes, and examining stability, temperature and $\mathrm{pH}$ effects.

\section{Acknowledgements}

We would like to acknowledge Andrew Wilkins for help with generating the biosensor schematics. We gratefully acknowledge financial support from the Centre of Applied Science for Health (CASH, IT Tallaght) which was funded by the Higher Education Authority under the Program for Research in Third Level Institutions (PRTLI) Cycle 4, Enterprise Ireland (TD/2008/107) and the European Regional Development Fund (ERDF), and Science Foundation Ireland (SFI 12/TIDA/I2308).

\section{References}

[1] J. Coyle, D. Price, M. DeLong, Alzheimer's disease: a disorder of cortical cholinergic innervation, Science 219 (4589) (1983) 1184-1190.

[2] J.O. Rinne, et al., A postmortem study of brain nicotinic receptors in Parkinson's and Alzheimer's disease, Brain Res. 547 (1) (1991) 155-158.

[3] M.G. Garguilo, A.C. Michael, Enzyme-modified electrodes for peroxide, choline, and acetylcholine, TrAC Trends Anal. Chem. 14 (4) (1995) 164-169.

[4] J.J. Burmeister, M. Palmer, G.A. Gerhardt, Ceramic-based multisite microelectrode array for rapid choline measures in brain tissue, Anal. Chim. Acta 481 (1) (2003) 65-74

[5] V. Parikh, et al., Rapid assessment of in-vivo cholinergic transmission by amperometric detection of changes in extracellular choline levels, Eur. J. Neurosci. 20 (6) (2004) 1545-1554.

[6] M. Wang, et al., Improved temporal resolution for in vivo microdialysis by using segmented flow, Anal. Chem. 80 (14) (2008) 5607-5615.

[7] A. Hekmat, et al., Effects of pH on the activity and structure of choline oxidase from Alcaligenes species, Acta Biochim. Pol. 55 (3) (2008) 549-557.

[8] B.M. Dixon, J.P. Lowry, R.D. O'Neill, Characterization in vitro and in vivo of the oxygen dependence of an enzyme/polymer biosensor for monitoring brain glucose, J. Neurosci. Methods 119 (2) (2002) 135-142.

[9] K.L. Baker, F.B. Bolger, J.P. Lowry, A microelectrochemical biosensor for real-time in vivo monitoring of brain extracellular choline, Analyst 140 (11) (2015) 3738-3745

[10] F.B. Bolger, R. Bennett, J.P. Lowry, An in-vitro characterisation comparing carbon paste and Pt microelectrodes for real-time detection of brain tissue oxygen, Analyst 136 (19) (2011) 4028-4035.

[11] J. Kealy, R. Bennett, J.P. Lowry, Simultaneous recording of hippocampal oxygen and glucose in real time using constant potential amperometry in the freely-moving rat, J. Neurosci. Methods 215 (1) (2013) 110-120.

[12] M.G. Garguilo, A.C. Michael, Quantitation of choline in the extracellular fluid of brain tissue with amperometric microsensors, Anal. Chem. 66 (17) (1994) 2621-2629.

[13] S. Ding, D. Shan, Y. Sun, Bioelectrochemical response of a choline biosensor fabricated by using polyaniline, Sci. China Ser. B: Chem. 52 (12) (2009) 2275-2280.

[14] P. Bernfeld, J. Wan, Antigens and enzymes made insoluble by entrapping them into lattices of synthetic polymers, Science 142 (3593) (1963) 678-679.

[15] K.B. O'Brien, et al., Development and characterization in-oitro of a catalase-based biosensor for hydrogen peroxide monitoring, Biosens. Bioelectron. 22 (12) (2007) 2994-3000.

[16] K.B. O'Brien, et al., Development and characterization in-oitro of a catalase-based biosensor for hydrogen peroxide monitoring, Biosens. Bioelectron. 22 (12) (2007) 2994-3000.

[17] S.A. Rothwell, S.J. Killoran, R.D. O’Neill, Enzyme immobilization strategies and electropolymerization conditions to control sensitivity and selectivity parameters of a polymer-enzyme composite glucose biosensor, Sensors 10 (7) (2010) 6439-6462.

[18] K.M. Mitchell, Acetylcholine and choline amperometric enzyme sensors characterized in vitro and in vivo, Anal. Chem. 76 (4) (2004) 1098-1106.

[19] A.L. Hart, W.A. Collier, Stability and function of screen printed electrodes, based on cholinesterase, stabilised by a co-polymer/sugar alcohol mixture, Sens. Actuators B 53 (1-2) (1998) 111-115.

[20] K. Reybier, et al., The use of polyethyleneimine for fabrication of potentiometric cholinesterase biosensors, Talanta 56 (6) (2002) 1015-1020.

[21] C.P. McMahon, et al., The efficiency of immobilised glutamate oxidase decreases with surface enzyme loading: an electrostatic effect, and reversal by a polycation significantly enhances biosensor sensitivity, Analyst 131 (1) (2006) 68-72.

[22] M.M. Andersson, R. Hatti-Kaul, Protein stabilising effect of polyethyleneimine, J. Biotechnol. 72 (1-2) (1999) 21-31.

[23] J. Jezkova, et al., Stabilization of an osmium bis-bipyridyl polymer-modified carbon paste amperometric glucose biosensor using polyethyleneimine, Electroanalysis 9 (13) (1997) 978-984.

[24] C.P. McMahon, et al., Oxygen tolerance of an implantable polymer/enzyme composite glutamate biosensor displaying polycation-enhanced substrate sensitivity, Biosens. Bioelectron. 22 (7) (2007) 1466-1473.

[25] D.B. Holt, et al., Fabrication of a capillary immunosensor in polymethyl methacrylate, Biosens. Bioelectron. 17 (1-2) (2002) 95-103.

[26] B.J. Doleman, N.S. Lewis, Comparison of odor detection thresholds and odor discriminablities of a conducting polymer composite electronic nose versus mammalian olfaction, Sens. Actuators B 72 (1) (2001) 41-50.

[27] B.M. Dixon, J.P. Lowry, R.D. O’Neill, Characterization in-vitro and in-vivo of the oxygen dependence of an enzyme/polymer biosensor for monitoring brain glucose, J. Neurosci. Methods 119 (2) (2002) 135-142.

[28] C.P. McMahon, et al., Control of the oxygen dependence of an implantable polymer/enzyme composite biosensor for glutamate, Anal. Chem. 78 (7) (2006) 2352-2359.

[29] C.P. McMahon, et al., Oxygen tolerance of an implantable polymer/enzyme composite glutamate biosensor displaying polycation-enhanced substrate sensitivity, Biosens. Bioelectron. 22 (7) (2007) 1466-1473.

[30] F.B. Bolger, et al., Characterisation of carbon paste electrodes for real-time amperometric monitoring of brain tissue oxygen, J. Neurosci. Methods 195 (2) (2011) 135-142. 
[31] F.B. Bolger, J.P. Lowry, Brain tissue oxygen: in-vivo monitoring with carbon paste electrodes, Sensors 5 (11) (2005) 473-487.

[32] K. Di Gleria, et al., Homogeneous ferrocene-mediated amperometric immunoassay, Anal. Chem. 58 (6) (1986) 1203-1205.

[33] B.A. Gregg, A. Heller, Redox polymer films containing enzymes. 1. A redox-conducting epoxy cement: synthesis, characterization, and electrocatalytic oxidation of hydroquinone, J. Phys. Chem. 95 (15) (1991) 5970-5975.

[34] M.G. Garguilo, A.C. Michael, An enzyme-modified microelectrode that detects choline injected locally into brain tissue, J. Am. Chem. Soc. 115 (25) (1993) $12218-12219$.

[35] S.K. Beh, G.J. Moody, J.D.R. Thomas, Studies on enzyme electrodes with ferrocene and carbon paste bound with cellulose triacetate, Analyst 116 (5) (1991) 459-462.

[36] N. Martens, E.A.H. Hall, Model for an immobilized oxidase enzyme electrode in the presence of two oxidants, Anal. Chem. 66 (17) (1994) 2763-2770.

[37] J. Wang, F. Lu, Oxygen-rich oxidase enzyme electrodes for operation in oxygen-free solutions, J. Am. Chem. Soc. 120 (5) (1998) 1048-1050.

[38] J. Wang, L. Chen, M.-P. Chatrathi, Evaluation of different fluorocarbon oils for their internal oxygen supply in glucose microsensors operated under oxygen-deficit conditions, Anal. Chim. Acta 411 (1-2) (2000) 187-192.

[39] M. Martín, et al., The use of fluorocarbons to mitigate the oxygen dependence of glucose microbiosensors for neuroscience applications, J. Electrochem. Soc. 161 (10) (2014) H689-H695.

[40] Y.G. Chirkov, V.I. Rostokin, Active layer of the oxygen cathode in a fuel cell with Nafion and platinum: the role played by the diffusion and ohmic restrictions and the selection of the working thickness of the active layer, Russ. J. Electrochem. 43 (7) (2007) 787-794.

[41] M. Schalenbach, et al., Gas permeation through nafion. part 1: measurements, J. Phys. Chem. C 119 (45) (2015) 25145-25155.

[42] M.G. Garguilo, A.C. Michael, Amperometric microsensors for monitoring choline in the extracellular fluid of brain, J. Neurosci. Methods 70 (1) (1996) $73-82$.

[43] R.D. O'Neill, The measurement of brain ascorbate In vivo and its link with excitatory amino acid neurotransmission, in: A.A. Boulton, G.B. Baker, R.N. Adams (Eds.), Voltammetric Methods in Brain Systems, Humana Press, Totowa, NJ, 1995, pp. 221-268.

[44] M. Miele, M. Fillenz, In-vivo determination of extracellular brain ascorbate, J. Neurosci. Methods 70 (1) (1996) 15-19.
[45] J.P. Lowry, et al., Characterization of glucose oxidase-modified poly(phenylenediamine)-coated electrodes in-vitro and in-vivo: homogeneous interference by ascorbic acid in hydrogen peroxide detection, Anal. Chem. 66 (10) (1994) 1754-1761.

[46] C.P. McMahon, R.D. O'Neill, Polymer-enzyme composite biosensor with high glutamate sensitivity and low oxygen dependence, Anal. Chem. 77 (4) (2005) 1196-1199.

[47] J.P. Lowry, R.D. O’Neill, Partial characterization in-vitro of glucose oxidase-modified poly(phenylenediamine)-coated electrodes for neurochemical analysis in-vivo, Electroanalysis 6 (1994) 369-379.

[48] J.D. Craig, R.D. O'Neill, Electrosynthesis and permselective characterisation of phenol-based polymers for biosensor applications, Anal. Chim. Acta 495 (1-2) (2003) 33-43.

\section{Biographies}

Keeley L. Baker, PhD received her BSc in Chemistry from National University of Ireland Maynooth (NUIM) in 2009. She received her Ph.D in Bioanalytical Chemistry from NUIM under the direction of Prof. John Lowry and Dr. Fiachra Bolger in 2013.

Fiachra B. Bolger, PhD received his BSc in Chemistry from National University of Ireland Maynooth (NUIM). He received his Ph.D in Bioanalytical Chemistry from NUIM under the direction of Prof. John Lowry.

John P. Lowry received his BSc in Chemistry from University College Dublin (UCD) in 1988. He received his Ph.D in Bioelectroanalytical Chemistry from UCD under the direction of Prof. Robert D. O'Neill in 1992. Prior to his first academic appointment he was a Marie Curie Fellow at the University of Oxford where he worked in the University Laboratory of Physiology with Dr. Marianne Fillenz. He was appointed as a University Lecturer in Analytical Chemistry at the National University of Ireland, Maynooth (NUIM), in 1998. In 2004 he became a lecturer in pharmacology at the Conway Institute UCD and returned to Maynooth University in 2006 to take up the Chair of Chemistry. His research interests are in the area of bioanalysis particularly in the development, characterisation and application of sensor and biosensor systems for in vivo neurochemical monitoring. 